\title{
Evaluation of Plasma Nucleosome Concentrations In Healthy Dogs And Dogs With A Variety of Common Cancers.
}

\section{Wilson-Robles, Heather ( $\sim$ HWilson@cvm.tamu.edu )}

Volition America \& Volition Veterinary Diagnostic Development

Bygott, Thomas

Volition Diagnostics UK Ltd

Kelly, Theresa Kathleen

Volition America \& Volition Veterinary Diagnostic Development

Miller, Tasha

Texas A\&M University

Miller, Pamela

Texas A\&M University

Matsushita, Matthew

Texas A\&M University

Terrell, Jason

Volition America \& Volition Veterinary Diagnostic Development

Bougoussa, Mhammed

Belgian Volition SRL

Butera, Thomas

Volition America \& Volition Veterinary Diagnostic Development

\section{Research Article}

Keywords: Nucleosome, histone, H3.1, canine, cancer, osteosarcoma, mast cell tumor, histiocytic sarcoma, melanoma, soft tissue sarcoma

Posted Date: December 29th, 2021

DOI: https://doi.org/10.21203/rs.3.rs-1193590/v1

License: (c) (i) This work is licensed under a Creative Commons Attribution 4.0 International License.

Read Full License 


\section{Abstract}

Background: Cell free DNA, in the form of nucleosomes, is released into circulation during apoptosis and necrosis in a variety of diseases. They are small fragments of chromosomes that are composed of DNA wrapped around a histone core made of four duplicate histone proteins forming an octamer. The nucleosome compartment is a relatively uninvestigated area of circulating tumor biomarkers in dogs. The objectives of this study were to quantify and better characterize nucleosomes in 528 dogs with various common malignancies and 134 healthy dogs.

Results: The sensitivity of elevated circulating nucleosome concentrations for the detection of cancer in all dogs was $49.8 \%$ with a specificity of $97 \%$ with an area under the curve of $68.74 \%$. The top 4 malignancies detected by the test included lymphoma, hemangiosarcoma, histiocytic sarcoma and malignant melanoma. The malignancies least likely to be detected were soft tissue sarcomas and mast cell tumors.

Conclusions: A variety of tumor types may cause elevated nucleosome concentations in dogs. Tumors of hematopoietic origin are most likely to cause elevations and local tumors such as soft tissue sarcomas are least likely to cause elevations in plasma nucleosome concentrations.

\section{Background:}

Biomarkers for the early detection of cancer have revolutionized cancer screening in healthy and high-risk human populations. These cancer screening tests enable enhanced opportunities for earlier treatment and higher cure rates in a variety of cancers(1). Tumor specific markers such as prostate-specific antigen (PSA), carcinoembryonic antigen (CEA), CA-15.3, and CA-27.29 have allowed for quick, non-invasive and inexpensive screening in large at risk populations in human medicine. Unfortunately, these types of assays are rare in veterinary medicine.

Many platforms have been utilized to assess blood based biomarkers for cancer. Traditionally, enzymelinked immunosorbent assays (ELISAs) or polymerase chain reactions (PCR) have been utilized to detect specific biomarkers. However, cancers are multifactorial and very few cancers share biomarker expression between them or even within the same histology. Whole genome sequencing can cast a wider net to detect a variety of biomarkers in the blood, but this approach is costly and time consuming. ELISAs directed at a cancer surrogate, such as nucleosomes, can provide a rapid, cost effective and widely applicable approach for biomarker detection in the blood of both humans and veterinary patients.

Nucleosomes are small fragments of chromosomes released into the blood during cell death or white blood cell activation. These fragments consist of a histone octamer core with a short segment of DNA wrapped around it. Nucleosomes have demonstrated utility as epigenetic biomarkers for the detection and monitoring of a variety of human cancers including pancreatic, lung, and colorectal cancer (2-5). Furthermore, plasma nucleosome concentrations have been shown to be predictive of outcome in patients with breast cancer. A multivariate analysis of 92 patients with breast cancer showed 
preoperative plasma nucleosome concentrations were as consistent as hormone receptor (HER2) status, lymph node status and tumor grade in predicting disease free survival(6). Plasma nucleosome levels can also predict response to therapy in patients with non-small cell lung cancer (NSCLC). In a study of 134 patients with advanced NSCLC, treated with chemotherapy, those with significant decreases in nucleosome concentrations after their first cycle of chemotherapy had significant improvement on imaging after their 3rd cycle. Those with insufficient decreases in plasma nucleosome concentrations were more likely to have stable or progressive disease and a significantly shorter progression free survival (7). Circulating plasma nucleosomes have also been used to identify genome- and exome-wide cancer specific mutations as well as longitudinal changes throughout the course of treatment that can be used to capture clonal evolution and indentify mechanisms of resistance $(8,9)$.

Plasma nucleosome concentrations are elevated in a variety of diseases, including cancer. Letendre et al published some of the earliest work describing the use of nucleosomes in dogs with trauma and sepsis in 2018 demonstrating that plasma nucleosome concentrations were postivitely correlated with a worse outcome in dogs with sepsis $(10,11)$. Additionally elevated plasma nucleosomes have been detected in cases of trauma, autoimmune disease and severe inflammation $(10,12-16)$. However, few studies exist defining the plasma nucleosome compartment in canine cancer. Recently, our group published data demonstrating elevated plasma nucleosome concentrations in dogs with lymphoma and hemangiosarcoma (17-19) and the goal of the current study was to expand upon that initial study to evaluate circulating nucleosome concentrations in dogs with a variety of common cancers. Understanding the which cancer types are associated with elevated nucleosome levels will not only expand the use of nucleosome screening but will also increase our understanding of nucleosomes and their role in cancer development and progression.

\section{Results:}

A total of 662 dogs were included in this study (528 dogs with cancer and 134 healthy dogs). The most common cancers evaluated included lymphoma (LSA; $n=126)$, Hemangiosarcoma (HSA; $n=77)$, osteosarcoma (OSA; $n=49)$, soft tissue sarcoma (STS; 51$)$, malignant melanoma ( $n=49)$, mast cell tumors (MCT; $n=126)$ and histiocytic sarcoma $(n=26)$ (Figure 1). A variety of carcinomas (mammary, pulmonary, hepatocellular, squamous cell, urothelial cell and anal sac carcinomas) as well as a few miscellaneous tumors (multiple myeloma, acute leukemias, insulinoma, nasal chondrosarcoma, sertoli cell tumor and others) were also evaluated. These cases are described here however, due to the low case numbers of any specific histology, they are not included in the statistical analyses reported here.

Of the 528 dogs with cancer, 244 were spayed females, 15 were intact females, 237 were neutered males and 30 were intact males. Gender was unknown for 2 of the dogs. These dogs ranged in age from $1-19$ years (median 9 years, mean 9.06 years) and ranged in weight from $5-74.5 \mathrm{~kg}$ (median $30.9 \mathrm{~kg}$, mean $29.63 \mathrm{~kg}$ ). The most commonly represented breeds included mixed breed dogs $(n=126)$, Labrador retriever $(n=58)$, golden retriever $(n=53)$ and boxers $(n=19)$. 
A total of 134 healthy dogs were recruited for this study ranging in age from 10 months to 14 years (median 6 years). There were 61 spayed femles, 4 intact females, 66 neutered males and 3 intact males. The most common breeds represented were mixed breed dogs $(n=28)$, Labrador retreivers $(n=15)$ and Australian cattle dogs $(n=10)$. Dogs were determined to be healthy based on results from a client questionnaire as well as a physical exam by the attending veterinarian. The median nucleosome concentration for all healthy dogs was $31.1 \mathrm{ng} / \mathrm{mL}$ (mean $32.07 \mathrm{ng} / \mathrm{mL}$, SEM 1.118). For a specificity of $100 \%$ the cut off for the healthy range was set at $67.5 \mathrm{ng} / \mathrm{mL}$ (nucleosome range for all healthy dogs was $6.33-67.42 \mathrm{ng} / \mathrm{mL})$.

When all cancer cases were considered together in comparison to healthy dogs the sensitivity of this assay was $49.8 \%$ with a specificity of $97 \%$ (Figure 1). A receiver operator characteristic (ROC) curve was generated and the AUC was determined to be $68.74 \%$ (Figure 2). The top 3 malignancies detected by the test included lymphoma, hemangiosarcoma and histiocytic sarcoma. The malignancies least likely to be detected using this assay were soft tissue sarcomas and mast cell tumors.

\section{H3.1 Nucleosome Levels Across Multiple Cancer Types}

For the purposes of group comparisons, a single specificity of $97 \%$ was chosen and the sensitivity was then calculated using this parameter. The cancers with the highest sensitivities were lymphoma (76.98\%), hemangiosarcoma (81.82\%), visceral histiocytic sarcoma (61.9\%) and malignant oral melanoma (50\%). The cancers with the lowest sensitivity were cutaneous melanoma (0\%), Mast cell tumors $(19.05 \%$ all grades, grade $1: 33.33 \%$, grade $2: 11.49 \%$ and grade $3: 34.62 \%$ ) and primary bone histiocytic sarcomas (20\%) (Table 1). Just under 35\% (17/49) of the osteosarcoma cases demonstrated elevated plasma nucleosome concentrations at diagnosis. Interestingly, the dog with the highest plasma nucleosome concentration in the osteosarcoma group $(446 \mathrm{ng} / \mathrm{mL})$ was the one dog that presented with metastatic disease at diagnosis (Tables 1 and 2).

When considering the plasma nucleosome concentrations of dogs with a variety of carcinomas, eight of the sixteen cases had elevated levels at the time of diagnosis. Two of the three of the anal sac apocrine gland adenocarcinomas had elevated plasma nucleosome levels $(101.2 \mathrm{ng} / \mathrm{mL}, 816.2 \mathrm{ng} / \mathrm{mL}$, and 33.8 $\mathrm{ng} / \mathrm{mL}$ ). Two of the three urothelial cell carcinoma cases had elevated plasma nucleosome concentrations at the time of diagnosis $(162.3 \mathrm{ng} / \mathrm{mL}, 129.6 \mathrm{ng} / \mathrm{mL}$ and $16.7 \mathrm{ng} / \mathrm{mL})$. There were 5 dogs with sinonasal or oral carcinomas in this study. Two of the $5 \mathrm{had}$ elevated plasma nucleosome concentrations at the time of diagnosis $(101.8 \mathrm{ng} / \mathrm{mL}, 110.7 \mathrm{ng} / \mathrm{mL}, 53.5 \mathrm{ng} / \mathrm{mL}, 42.1 \mathrm{ng} / \mathrm{mL}, 8.2$ $\mathrm{ng} / \mathrm{mL}$ ). The two dogs with elevated levels were both diagnosed with oral squamous cell carcinomas. There were 3 dogs in this group with primary lung carcinomas, all of which had elevated nucleosome concentrations $(76.2 \mathrm{ng} / \mathrm{mL}, 94.4 \mathrm{ng} / \mathrm{mL}, 548.5 \mathrm{ng} / \mathrm{mL})$. One dog with mammary carcinoma and one dog with hepatocellluar carcinoma were also included in this study. Neither dog had elevated nucleosome concentrations ( $25.0 \mathrm{ng} / \mathrm{mL}$ and $28.5 \mathrm{ng} / \mathrm{mL}$ respectively).

Several other tumors were included in this study as well. Both dogs with acute leukemia had elevated plasma nucleosome concentrations (lymphoblastic leukemia - $262.8 \mathrm{ng} / \mathrm{mL}$, myeloblastic leukemia 
$423.5 \mathrm{ng} / \mathrm{mL}$ ). We also saw elevated plasma nucleosome concentrations in a dog with a sertoli cell tumor $(118 \mathrm{ng} / \mathrm{mL})$, a dog with a pharyngeal sarcoma $(135.4 \mathrm{ng} / \mathrm{mL})$, a dog with nasal chondrosarcoma $(109.25 \mathrm{ng} / \mathrm{mL})$ and a dog with multiple myeloma $(71.7 \mathrm{ng} / \mathrm{mL})$. There were three cases that did not have elevated plasma nucleosome concentrations including a dog with an undifferentiated splenic sarcoma $(16.4 \mathrm{ng} / \mathrm{mL})$, a dog with insulinoma $(8.5 \mathrm{ng} / \mathrm{mL})$ and a dog with multilobular osteochondrosarcoma (20.3 ng/mL).

Table 1

Summary of the Sensitivity and specificity of plasma nucleosomes to detect a variety of canine cancers.

\begin{tabular}{|llllll|}
\hline Cancer Histology & $\begin{array}{l}\text { Number of } \\
\text { cases }\end{array}$ & $\begin{array}{l}\text { Elevated Nu.Q® } \\
\text { level }\end{array}$ & Sensitivity & Specificity & AUC \\
\hline All Cancers & 504 & $251 / 504$ & $49.8 \%$ & $97 \%$ & $68.74 \%$ \\
\hline Lymphoma & 126 & $97 / 126$ & $76.98 \%$ & $97 \%$ & $87.83 \%$ \\
\hline Hemangiosarcoma & 77 & $63 / 77$ & $81.82 \%$ & $97 \%$ & $91.74 \%$ \\
\hline Histiocytic Sarcoma & 26 & $14 / 26$ & $53.85 \%$ & $97 \%$ & $83.01 \%$ \\
\hline Hist Sarc - bone & 5 & $1 / 5$ & $20 \%$ & $97 \%$ & $81.04 \%$ \\
\hline Hist Sarc Visceral & 21 & $13 / 21$ & $61.9 \%$ & $97 \%$ & $83.48 \%$ \\
\hline Melanoma (all) & 49 & $21 / 49$ & $42.86 \%$ & $97 \%$ & $70.36 \%$ \\
\hline Melanoma (20) & 42 & $21 / 42$ & $50 \%$ & $97 \%$ & $75.05 \%$ \\
\hline $\begin{array}{l}\text { Melanoma } \\
\text { (cutaneous) }\end{array}$ & 7 & $0 / 7$ & $0 \%$ & $97 \%$ & $42.22 \%$ \\
\hline Mast Cell Tumor & 126 & $24 / 126$ & $19.05 \%$ & $97 \%$ & $43.68 \%$ \\
\hline Grade 1 MCT & 9 & $3 / 9$ & $33.33 \%$ & $97 \%$ & $44.1 \%$ \\
\hline Grade 2 MCT & 87 & $10 / 87$ & $11.49 \%$ & $97 \%$ & $11.49 \%$ \\
\hline Grade 3 MCT & 26 & $9 / 26$ & $34.62 \%$ & $97 \%$ & $60.7 \%$ \\
\hline Osteosarcoma & 49 & $17 / 49$ & $34.69 \%$ & $97 \%$ & $60.17 \%$ \\
\hline Soft tissue sarcoma & 51 & $15 / 51$ & $29.41 \%$ & $97 \%$ & $48.19 \%$ \\
\hline
\end{tabular}


Table 2

Nucleosome concentrations by histology

\begin{tabular}{|llllllll|}
\hline H3.1 (ng/mL) & $\mathbf{n}$ & Minimum & Maximum & Median & Mean & SEM & $\mathbf{p}$ \\
\hline HSA & 77 & 6.54 & 1956.90 & 198.30 & 414.62 & 53.14 & $\mathbf{6 . 3 3 E}-24$ \\
\hline LSA & 126 & 0.10 & 6544.00 & 211.05 & 570.87 & 90.85 & $\mathbf{5 . 6 2 E}-26$ \\
\hline OSA & 49 & 0.10 & 446.00 & 43.20 & 72.70 & 12.85 & $\mathbf{0 . 0 3 5 4}$ \\
\hline STS & 51 & 0.10 & 2500.00 & 25.09 & 200.07 & 82.24 & 0.7043 \\
\hline MCT & 126 & 0.10 & 969.45 & 24.65 & 64.21 & 11.67 & 0.0784 \\
\hline MCT Grade unk & 4 & 11.02 & 174.58 & 83.77 & 88.29 & 43.03 & 0.8341 \\
\hline MCT Grade 3 & 26 & 8.39 & 969.45 & 38.21 & 149.74 & 49.46 & 0.0849 \\
\hline MCT Grade 2 & 87 & 3.10 & 337.23 & 22.67 & 37.52 & 5.85 & $\mathbf{0 . 0 0 2 9}$ \\
\hline MCT Grade 1 & 9 & 0.10 & 313.81 & 18.26 & 64.41 & 33.04 & 0.5579 \\
\hline Melanoma & 49 & 7.28 & 1234.50 & 44.59 & 115.73 & 27.72 & $\mathbf{2 . 5 3 E - 0 5}$ \\
\hline Histiocytic sarcoma & 26 & 21.81 & 1800.00 & 69.59 & 261.22 & 81.31 & $\mathbf{1 . 0 6 E - 0 7}$ \\
\hline Healthy & 134 & 6.33 & 67.42 & 31.10 & 32.07 & 1.12 & 1 \\
\hline
\end{tabular}

Detailed evaluations of plasma nucleosome concentrations in dogs with lymphoma and hemangiosarcoma by stage, location or phenotype have been described elsewhere $(18,19)$. For the cases with melanoma there were two main groups. The first represents cutaneous melanoma of haired skin which are often considered benign $(n=7)$ and those in the oral cavity $(n=42)$ which are often malignant. The median plasma nucleosome concentration for the dogs with cutaneous melanoma was $24.8 \mathrm{ng} / \mathrm{mL}$ (mean $27.6 \mathrm{ng} / \mathrm{mL}$, range 7.3-43.9) and the median size of these tumors was $3 \mathrm{~cm}$ (mean $4.7 \mathrm{~cm}$, range 1.8-12 cm) (Figure 3). The median plasma nucleosome concentration for dogs with oral melanoma was $60.0 \mathrm{ng} / \mathrm{mL}$ (mean 130.422, range 14.0-1234.5 ng/mL) and the median size of these tumors was $4 \mathrm{~cm}$ (mean $4.5 \mathrm{ng} / \mathrm{mL}$, range $1.5-15 \mathrm{~cm}$ )(Figure 3). Mitotic index was not available for evaluation.

Mast cell tumors were divided by recorded grade. All tumors in the DCTD biobank were categorized according to the Patnaik 3 tier grading scheme. Grade 3 tumors had the highest mean plasma nucleosome concentrations ( 38.2 and $149.7 \mathrm{ng} / \mathrm{mL}$ respectively) followed by grade 1 tumors. Grade 2 tumors had the lowest nucleosome concentrations of the group (Figure 4,Table 2). Follow up information regarding overall survival was not available for the dogs with elevated plasma nucleosome concentrations.

Overall, visceral histiocytic sarcomas as a group had elevated plasma nucleosome concentrations similar to lymphoma and hemangiosarcoma. Those cases involving the spleen had the highest median plasma nucleosome concentrations. Histiocytic sarcomas involving the bone had the lowest median plasma nucleosome concentrations (Figure 5)(19). 


\section{Discussion:}

Nucleosome levels were evaluated in plasma samples from patients with seven of the most common canine cancers. The dogs with the most frequent elevations in plasma nucleosome concentrations were dogs with hemangiosarcoma and lymphoma, consistent with previous publications $(18,19)$. Other cases with frequent elevations in plasma nucleosome concentrations include those with histiocytic sarcoma and oral malignant melanoma.

Not surprisingly, the majority of dogs with visceral disease had elevated plasma nucleosome concentrations. However, only one of the cases with primary osseous histiocytic sarcoma had elevated plasma nucleosome concentrations. This is in contrast to the fact that $100 \%$ of primary osseous hemangiosarcomas had elevated plasma nucleosome concentrations in one study (19). In this study, only $34.7 \%$ of dogs with osteosarcoma had elevated plasma nucleosome concentrations at diagnosis as well. Though osteosarcoma in dogs is often an aggressive tumor with a rapid growth rate, the local nature of this disease at the time of diagnosis is most likely part of the reason why so few of these cases have elevated nucleosome concentrations. Interestingly the dog diagnosed with osteosarcoma that had the highest elevations in nucleosome concentrations $(446 \mathrm{ng} / \mathrm{mL})$ was the only dog in the osteosarcoma that had evidence of metastatic disease, suggesting that as this disease becomes more systemic it may be more likely correlated with elevations in plasma nucleosome concentrations. Additional cases of metastatic disease are needed to determine the value of plasma nucleosome concentrations as a predictor of metastatic disease in dogs with osteosarcoma as well as other cancers.

Overall, the assay was only able to correctly predict $19 \%$ of the cases with mast cell tumors. Dogs with grade 1 tumors were detected $33 \%$ of the time and dogs with high grade tumors were detected $34 \%$ of the time, whereas grade 2 tumors were only detected $11.5 \%$ of the time. It is unclear from the patient information available why there is a difference between grade 2 tumors and the other grades. The high grade mast cell tumors had a higher mean nucleosome concentration than the other grades. Unfortunately, we do not have the outcome data associated with this group and cannot determine whether those cases with elevated plasma nucleosome concentrations have a better or worse prognosis.

Interestingly, in this cohort of samples none of the benign cutaneous melanomas had elevated plasma nucleosome concentrations, while just over half of the malignant oral melanomas demonstrated elevated plasma nucleosome concentrations. This finding highlights the possibility of using nucleosome levels to discriminate between benign and malignant processes in dogs with melanoma. All of the malignant melanomas in this study were oral in origin. Additional cases from other commonly malignant locations such as the ungual process or the perianal region need to be evaluated to determine if this trend holds true for all cases of malignant melanoma.

Similarly to what has been noted in humans, the three cases with primary lung carcinomas had elevations in plasma nucleosome concentrations (21-24). Two of the three dogs with anal sac tumors also had significant elevations in plasma nucleosome concentrations. The one dog with a plasma 
nucleosome concentration over $800 \mathrm{ng} / \mathrm{mL}$ had stage I disease that was treated with surgery demonstrating that this may also be a useful tool for dogs with early stage anal sac neoplasia. Two of the 5 cases of oral/sinonasal carcinomas had elevated nucleosome concentrations. Given the local nature of these diseases, we did not expect to see elevations in any of these cases. However, both of these cases were squamous cell carcinomas. These tumors tend to have a large inflammatory component that may be responsible for the elevations of plasma nucleosome concentrations seen here. It was also surprising to see elevated nucleosome concentrations in $2 / 3$ of the cases with urothelial carcinoma. Again, these cases had local disease only. It was also not surprising to see elevated nucleosome conccentrations in the one case of multiple myeloma given that it is a systemic tumor and a tumor of plasma cells. However, the fact that the stage III nasal chondrosarcoma, which tends to be slow growing, displayed elevations in plasma nucleosomes was unexpected. Local inflammation at the site of the tumor may play a role here and additional cases of sinonasal tumors as well as non-melanoma oral malignancies are being enrolled. Several cases did not have elevated plasma nucleosome concentrations. These include the cases of insulinoma, mammary carcinoma, MLO, undifferentiated splenic sarcoma and hepatocellular carcinoma did not demonstrate elevations in plasma nucleosome concentrations. This may be due to the low disease burden or the slow cellular turnover rate associated with most of these tumors. Additional cases of non-hemangiosarcoma splenic masses are being actively recruited to better understand these diseases.

\section{Conclusions And Future Directions:}

In summary, although the majority of information published regarding plasma nucleosome concentrations in dogs with cancer have been centered around lymphoma and hemangiosarcoma, this test is likely useful for detecting other forms of cancer as well. The test performs best for tumors that are systemic (higher metastatic rate) and for those that have a high cellular turnover rate. This is consistent with the observation that nucleosomes are released into the plasma at a higher rate when there is a rapid cellular turn over and high cellular death rates. This is also true for non-cancerous diseases including severe infections or inflammation. Additional studies are ongoing to determine how noncancerous concomitant diseases affect the plasma nucleosome compartment in dogs.

Evaluation of H3.1 plasma nucleosome concentrations using a low-cost simple ELISA test requiring low blood sample volume is not only useful as a cancer screening tool but may also provide additional information regarding the overall health and well being of companion dogs. This test is well positioned as a companion test to other wellness tests and has the potential to provide valuable additional information that can inform the clinical decision-making process.

\section{Methods:}

All animal studies were approved by the Texas A\&M University Institutional Animal Care and Use Committee (25 2019-0211 and AUP\# 2017-0350). A detailed characterization of the participants has been included in the results section. Canine plasma samples from the National Cancer Institute Division of 
Cancer Treatment and Diagnosis (NCI-DCTD) biorepository and from active patients or healthy volunteers at the Texas A\&M University Small Animal Teaching Hospital were purchased or collected with owner consent, respectively, for this study. A minimum of $0.5 \mathrm{~mL}$ of plasma was collected from each patient. Dogs were fasted for a minimum of 4 hours before blood collection. Samples were drawn from a peripheral or jugular vein into a K2 EDTA (lavender top) tube (BD Vacutainer, Franklin Lakes, NJ) and centrifuged at $3000 \mathrm{~g}$ for 10 minutes within 1 hour of collection (Clinical 100 centrifuge, VWR, Radnor, PA). The plasma samples were labeled and frozen at $-80 \mathrm{C}$ until samples were run in batches.

All samples were tested using the Nu.Q® Vet Cancer Screening test (Belgian Volition, SRL, Isnes, Belgium). This is an enzyme-linked immunosorbent assay (ELISA) that contains a capture antibody directed at histone 3.1 and a nucleosome specific detection antibody (26). Samples were thawed and allowed to come to room temperature for at least 30 minutes prior to analysis. Assays were performed according to the manufacturer's protocol. The plates were read on a plate reader at $450 \mathrm{~nm}$ (BioTek Synergy H1 plate reader, BioTek Instruments, Winooski, VT). The standard curve was linearized and fitted to a 4-parameter logistic curve using statistical software (Graphpad Prism Software, version 9, San Diego, CA, USA, www.graphpad.com). Descriptive statistics for the patient populations were performed using Microsoft Excel for Mac (v. 16.16.27, 2016). For data sets containing only two cohorts, such as the healthy controls versus all mast cell tumor cases, a Wilcoxon rank sum test was used to compare the medians of the data sets. For data sets where multiple conditions were compared, such as when all cancers were considered, a two-way ANOVA for repeat measures with a Tukey's multiple comparisons test was performed using GraphPad Prism Software version 9, San Diego, California USA, www.graphpad.com. Wilcoxon rank sum tests, ROC curves and specificity/sensitivity calculations were only performed on cancer groups for which there were at least 20 cases. These calculations were performed using R version 3.4 .3 and the pROC package $(27,28)$.

\section{Abbreviations:}

DNA: Deoxyribose Nucleic Acid

PSA: Prostate Specific Antigen

CEA: Carcinoembryonic Antigen

ELISA: Enzyme Linked Immunosorbent Assay

PCR: Polymerase Chain Reaction

NSCLC: Non Small Cell Lung Cancer

LSA: Lymphoma/Lymphosarcoma

OSA: Osteosarcoma 
STS: Soft tissue Sarcoma

MCT: Mast Cell Tumor

SEM: Standard Error of the Mean

ROC: Receiver Operator Characteristic Curve

NCI-DCTD: National Cancer Institute Division of Cancer Treatment and Diagnosis biorepository

EDTA: Ethylenediaminetetraacetic acid

g: Relative Centrifugal Force

C: Celcius

ANOVA: Analysis of Variance

\section{Declarations:}

Ethics approval and consent to participate:

All animal studies were approved by the Texas A\&M University Animal Care and Use Committee (AUP \#2017-0350). Client-owned animals were evaluated with the informed consent of the owner (AUP \#20190211).

\section{All methods were carried out in accordance with relevant guidelines and regulations.}

No humans were involved in this study.

No agents were administered to dogs in any way during this study.

Consent for publication:

Not applicable

Availability of data and materials

All relevant data are within the paper.

Competing interests

JT, TK, MB, TBu and TBy are employees of Volition Diagnostics UK Ltd \& Volition America, which have patents covering Nu.Q technology and are developers of Nu.QTM assays. Volition Veterinary is a joint venture between Belgian Volition and Texas A\&M University. HWR is a paid consultant of Volition Veterinary. TM, MM and JJ have no conflicts of interest to declare. Additional salary support for TM was 
provided by the Fred and Vola Palmer Chair in Comparative Oncology held by HWR. The Palmers did not play a role in the study design, data collection and analysis, decision to publish, or preparation of the manuscript and only provided financial support for the authors' salaries (TM). This does not alter our adherence to BMC policies on sharing data and materials.

\section{Funding}

Funding for materials and author salaries was provided by Belgian Volition SRL. HWR, MM and TM received salary from these funds. JT, TK, MB and TB are employees of Volition Diagnostics UK Ltd \& Volition America. The URL to the Volition website is: https://volition.com/. Some additional funding for HWR and TM salaries was provided by the Fred and Vola Palmer Chair of Comparative Oncology held by HWR.

\section{Authors' contributions}

Conceptualization: HWR

Data curation: HWR, TM, JJ, MM

Formal analysis: HWR, TK, TBy, MB

Funding acquisition: HWR, JT

Investigation: HWR, JJ, MM.

Methodology: HWR, TM.

Project administration: HWR, JJ, MM

Resources: HWR, TM, JJ, MB, JT, TK, TBy, MB, TBu.

Software: HWR, TBy, MB

Supervision: HWR

Validation: HWR, TM

Visualization: HMW

Writing - original draft: HMW

Writing - review \& editing: All

Authors Acknowledgements 
The authors would like to acknowledge the Fred and Vola Palmer Chair in Comparative Oncology for funding support of this work.

Authors' information (optional).

Not applicable.

\section{References:}

1. Schiffman JD, Fisher PG, Gibbs P. Early detection of cancer: past, present, and future. Am Soc Clin Oncol Educ Book. 2015:57-65.

2. Bauden $M$, Pamart $D$, Ansari $D$, Herzog $M$, Eccleston $M$, Micallef $J$, et al. Circulating nucleosomes as epigenetic biomarkers in pancreatic cancer. Clin Epigenetics. 2015;7:106.

3. Rahier JF, Druez A, Faugeras L, Martinet JP, Géhénot M, Josseaux E, et al. Circulating nucleosomes as new blood-based biomarkers for detection of colorectal cancer. Clin Epigenetics. 2017;9:53.

4. Rasmussen L, Christensen IJ, Herzog M, Micallef J, Nielsen HJ, Danish Collaborative Group on Early Detection of Colorectal C. Circulating cell-free nucleosomes as biomarkers for early detection of colorectal cancer. Oncotarget. 2018;9(12):10247-58.

5. Cristiano S, Leal A, Phallen J, Fiksel J, Adleff V, Bruhm DC, et al. Genome-wide cell-free DNA fragmentation in patients with cancer. Nature. 2019;570(7761):385-9.

6. Mego M, Kalavska K, Karaba M, Minarik G, Benca J, Sedlackova T, et al. Plasma Nucleosomes in Primary Breast Cancer. Cancers (Basel). 2020;12(9).

7. Kumar S, Guleria R, Singh V, Bharti AC, Mohan A, Das BC. Plasma nucleosome levels might predict response to therapy in patients with advanced non-small-cell lung cancer. Clin Lung Cancer. 2010;11(1):36-44.

8. Murtaza M, Caldas C. Nucleosome mapping in plasma DNA predicts cancer gene expression. Nat Genet. 2016;48(10):1105-6.

9. Ulz P, Thallinger GG, Auer M, Graf R, Kashofer K, Jahn SW, et al. Inferring expressed genes by wholegenome sequencing of plasma DNA. Nat Genet. 2016;48(10):1273-8.

10. Letendre JA, Goggs R. Determining prognosis in canine sepsis by bedside measurement of cell-free DNA and nucleosomes. J Vet Emerg Crit Care (San Antonio). 2018;28(6):503-11.

11. Letendre JA, Goggs R. Concentrations of Plasma Nucleosomes but Not Cell-Free DNA Are Prognostic in Dogs Following Trauma. Front Vet Sci. 2018;5:180.

12. Letendre JA, Goggs R. Measurement of plasma cell-free DNA concentrations in dogs with sepsis, trauma, and neoplasia. J Vet Emerg Crit Care (San Antonio). 2017;27(3):307-14.

13. Martiny P, Goggs R. Biomarker Guided Diagnosis of Septic Peritonitis in Dogs. Front Vet Sci. 2019;6:208.

14. Monestier M, Novick KE, Karam ET, Chabanne L, Monier JC, Rigal D. Autoantibodies to histone, DNA and nucleosome antigens in canine systemic lupus erythematosus. Clin Exp Immunol. 
1995;99(1):37-41.

15. Jeffery U, Kimura K, Gray R, Lueth P, Bellaire B, LeVine D. Dogs cast NETs too: Canine neutrophil extracellular traps in health and immune-mediated hemolytic anemia. Vet Immunol Immunopathol. 2015;168(3-4):262-8.

16. Krogh AKH, Lyngby JG, Bjørnvad CR, Nielsen LN. Presence of nucleosomes in plasma and increased thrombin generation in dogs with acute and chronic gastroenteropathies. Res Vet Sci. 2021;135:504-10.

17. Wilson-Robles H, Miller T, Jarvis J, Terrell J, Dewsbury N, Kelly T, et al. Evaluation of nucleosome concentrations in healthy dogs and dogs with cancer. PLoS One. 2020;15(8):e0236228.

18. Dolan C, Miller T, Jill J, Terrell J, Kelly TK, Bygott T, et al. Characterizing circulating nucleosomes in the plasma of dogs with lymphoma. BMC Vet Res. 2021;17(1):276.

19. Wilson-Robles H, Miller T, Jarvis J, Terrell J, Kelly TK, Bygott T, et al. Characterizing circulating nucleosomes in the plasma of dogs with hemangiosarcoma. BMC Vet Res. 2021;17(1):231.

20. Morales-Molina A, Gambera S, Leo A, Garcia-Castro J. Combination immunotherapy using G-CSF and oncolytic virotherapy reduces tumor growth in osteosarcoma. J Immunother Cancer. 2021;9(3).

21. Bowman RV, Yang IA, Semmler AB, Fong KM. Epigenetics of lung cancer. Respirology. 2006;11(4):355-65.

22. Holdenrieder S, Nagel D, Schalhorn A, Heinemann V, Wilkowski R, von Pawel J, et al. Clinical relevance of circulating nucleosomes in cancer. Ann N Y Acad Sci. 2008;1137:180-9.

23. Tufman A, Huber RM. Biological markers in lung cancer: A clinician's perspective. Cancer Biomark. 2010;6(3-4):123-35.

24. Wang K, Shan S, Wang S, Gu X, Zhou X, Ren T. HMGB1-containing nucleosome mediates chemotherapy-induced metastasis of human lung cancer. Biochem Biophys Res Commun. 2018;500(3):758-64.

25. Cedervall J, Dragomir A, Saupe F, Zhang Y, Arnlov J, Larsson E, et al. Pharmacological targeting of peptidylarginine deiminase 4 prevents cancer-associated kidney injury in mice. Oncoimmunology. 2017;6(8):e1320009.

26. Arimura Y, Ikura M, Fujita R, Noda M, Kobayashi W, Horikoshi N, et al. Cancer-associated mutations of histones H2B, H3.1 and H2A.Z.1 affect the structure and stability of the nucleosome. Nucleic Acids Res. 2018;46(19):10007-18.

27. Team RC. R: A language and environment for statistical computing. 2017.

28. Xavier Robin NT, Alexandre Hainard, et al.. pROC: an open-source package for R and S+ to analyze and compare ROC curves. BMC Bioinformatics. 2011;7(77).

\section{Figures}




\section{H3.1 Nucleosome Levels Across Multiple Cancer Types}

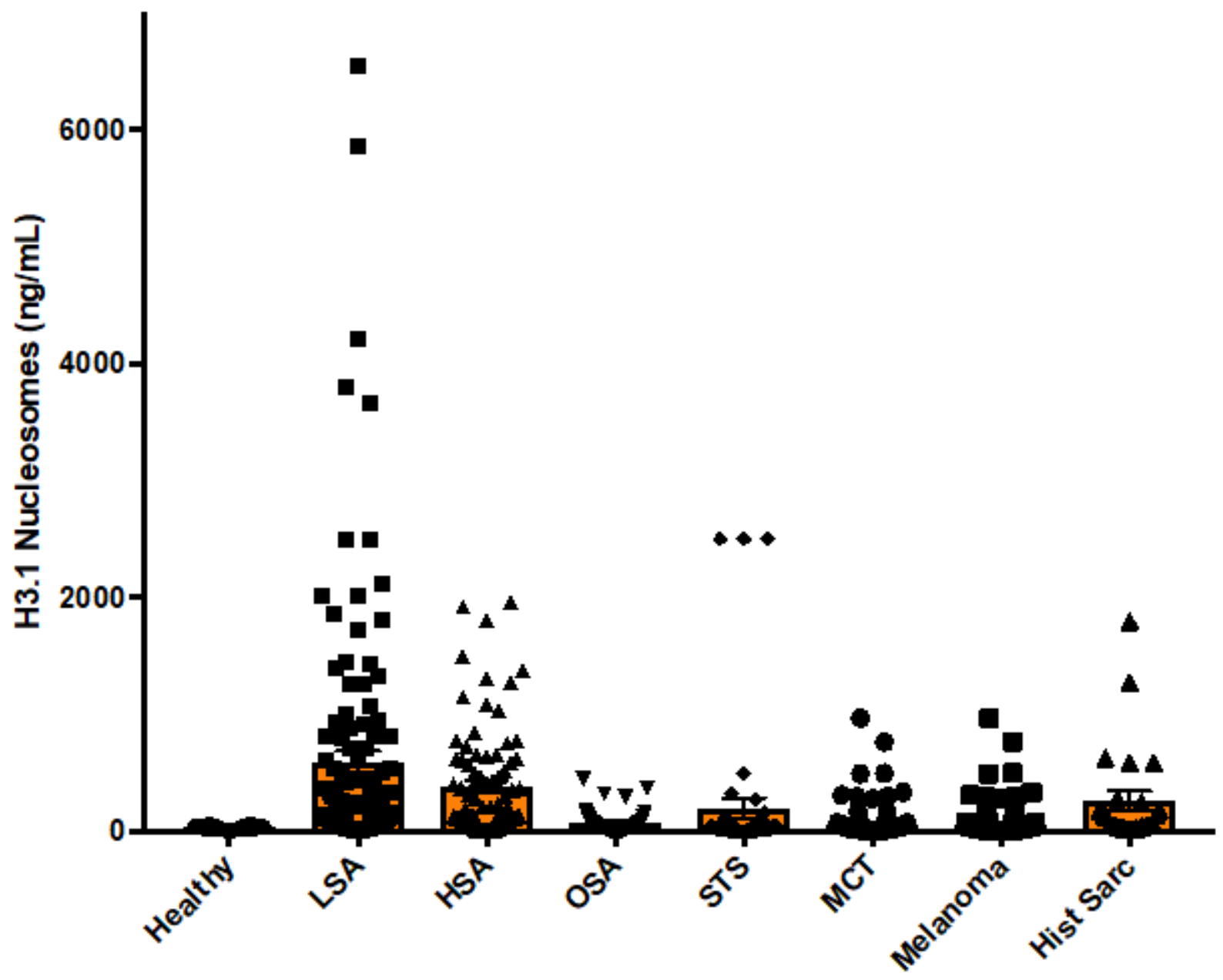

Figure 1

Box plot representing the various common cancers relative to the healthy cases. The y axis represents the plasma concentration of $\mathrm{H} 3.1$ nucleosomes in $\mathrm{ng} / \mathrm{mL}$ and the $\mathrm{x}$ axis represents the different malignancies evaluated. 


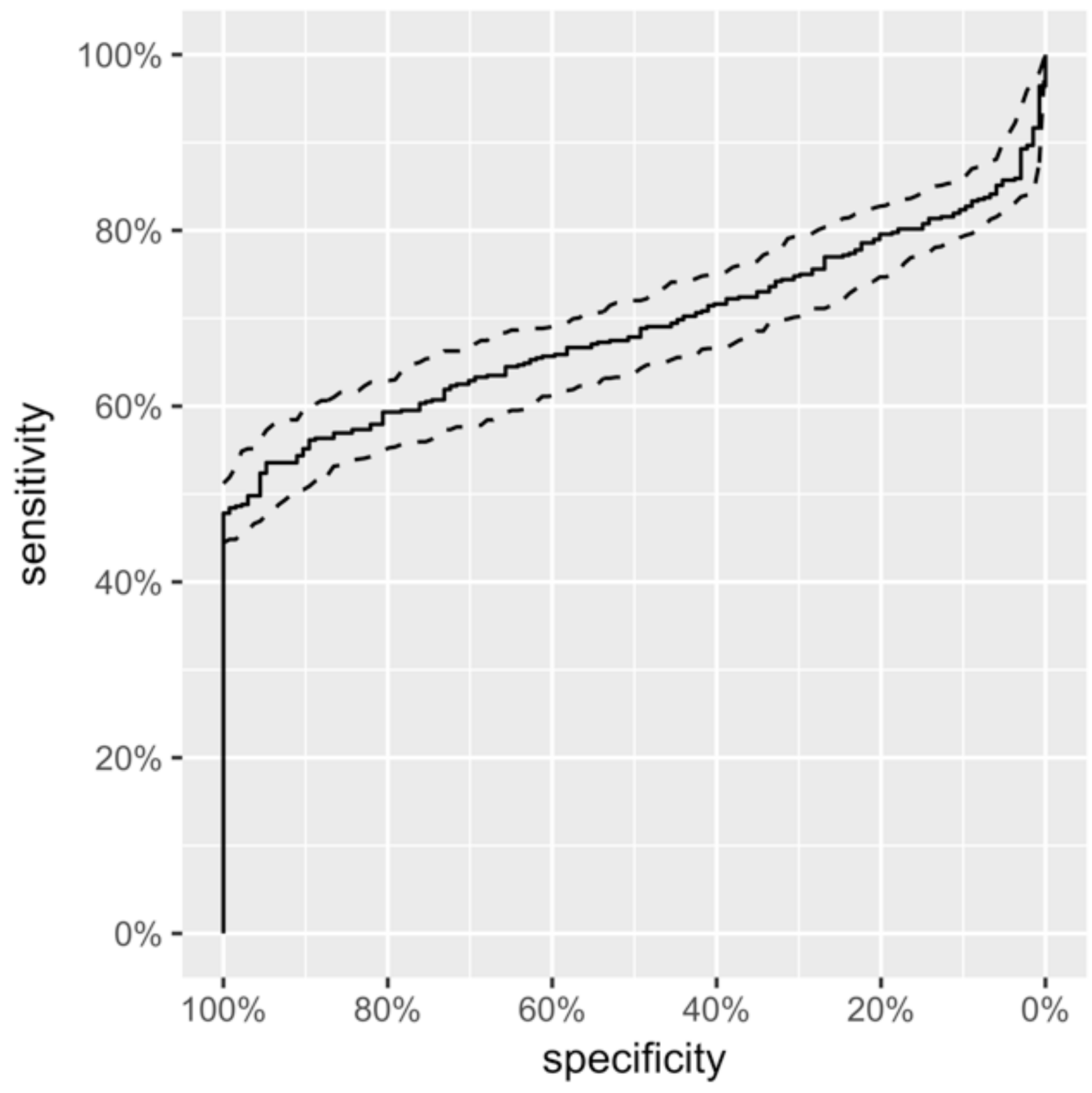

Figure 2

ROC curve demonstrating the AUC of $68.74 \%$ for all cancers when compared to healthy animals 


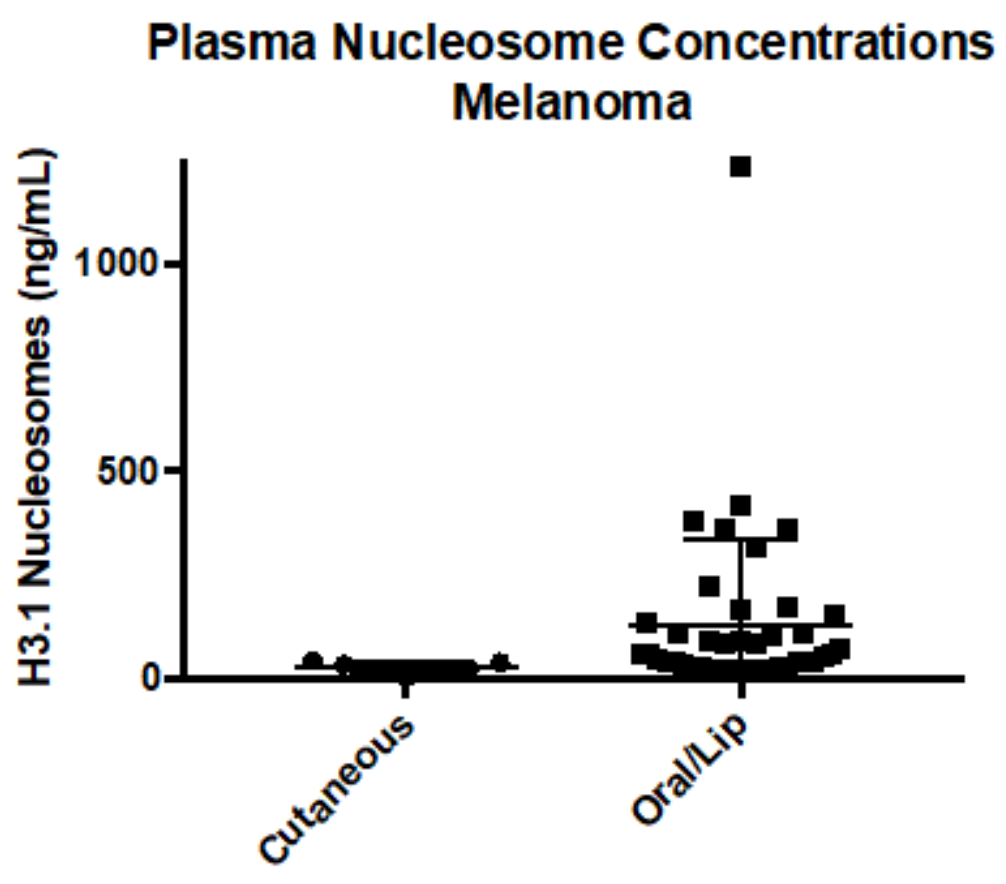

Figure 3

$\mathrm{H} 3.1$ plasma nucleosome concentrations $(\mathrm{ng} / \mathrm{mL})$ for melanoma cases by location.

\section{Plasma Nucleosome Concentrations}

\section{Mast Cell Tumors}

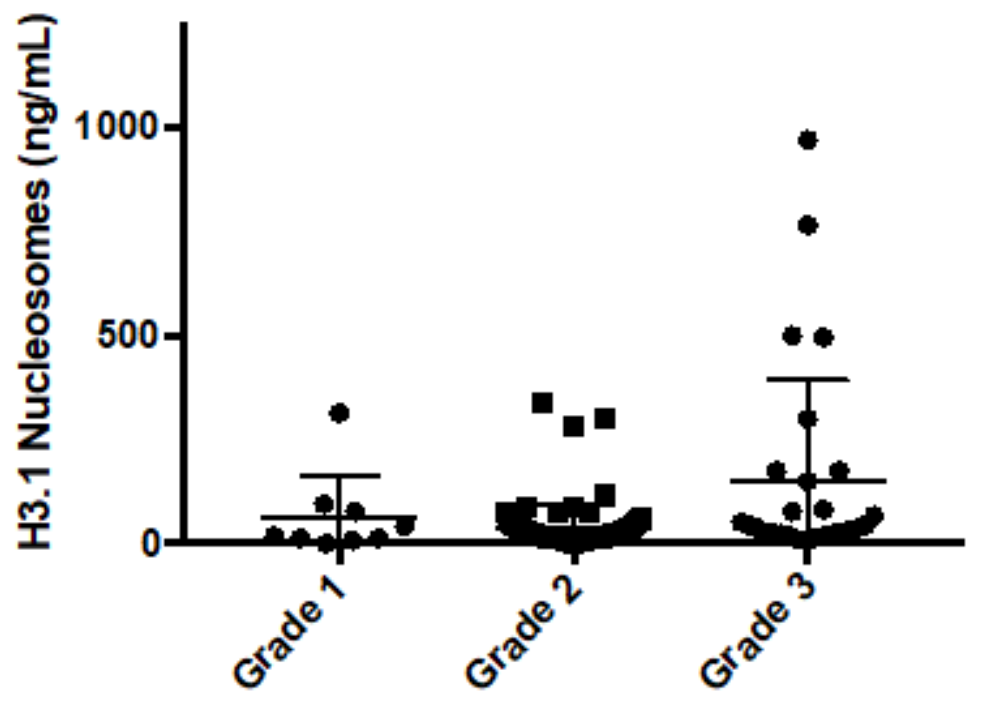

Figure 4

Plasma nucleosome concentrations from dogs with mast cell tumors according to grade. 


\section{Plasma Nucleosome Concentrations Histiocytic Sarcoma}

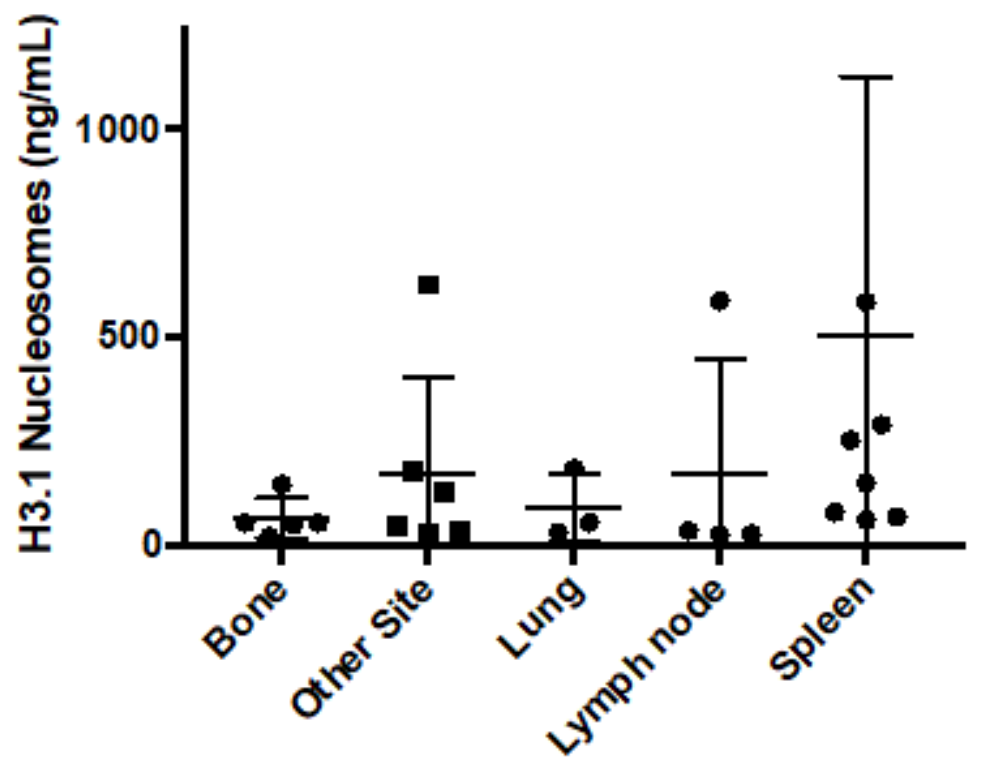

Figure 5

Plasma nucleosome concentrations Histiocytic Sarcoma 